\title{
Epstein-Barr virus-associated gastric carcinoma
}

INSERM

\section{Source}

INSERM. (1999). Orphanet: an online rare disease and orphan drug data base. EpsteinBarr virus-associated gastric carcinoma. ORPHA:313920

Epstein-Barr virus (EBV)-associated gastric carcinoma (EBVaGC) is a rare form of gastric carcinoma (seen in approximately $10 \%$ of cases) with a male predominance, characterized by a latent EBV infection in gastric carcinoma cells, diffuse-type histology, a proximal location (in the body and cardia of the stomach) and a relatively favorable prognosis. 